\title{
Triangular hysteresis loops in the spin-rotation region of orthoferrites
}

\author{
Ya.B. Bazaliy ${ }^{1,2}$ and L.T. Tsymbal ${ }^{3}$ \\ ${ }^{1}$ Institute of Magnetism of the National Academy of Sciences of Ukraine, 36 Vernadskogo Blvd., Kyiv 03143, Ukraine \\ ${ }^{2}$ University of South Carolina, Columbia SC 29208, USA \\ ${ }^{3}$ O. Galkin Donetsk Physics \& Technology Institute of the National Academy of Sciences of Ukraine \\ 72 R. Luxemburg Str., Donetsk 83114, Ukraine \\ E-mail: 1t_tsymbal@yahoo.com
}

Received January 22, 2010

\begin{abstract}
Suggested theory qualitatively explains the shapes of the hysteresis loops in orthoferrites within the temperature interval of the magnetic reorientation transition. Triangular loops result from the strong temperature dependence of both the magnetic moment and the magnetic domain wall structure.

PACS: 75.60.Ej Magnetization curves, hysteresis, Barkhausen and related effects;

75.60.Jk Magnetization reversal mechanisms;

75.50.Gg Ferrimagnetics;

75.30.Kz Magnetic phase boundaries (including classical and quantum magnetic transitions, metamagnetism, etc.).
\end{abstract}

Keywords: orthoferrites, domain wall structure, hysteresis loops.

Dedicated to V.G. Baryakhatar's anniversary. Sapientia superat mora.

The orthoferrites have a chemical composition $\mathrm{RFeO}_{3}$, where $\mathrm{R}$ is the rare-earth element. They are well studied magnetic materials. It is known for a long time [1] that for $\mathrm{R}=\mathrm{Er}, \mathrm{Tm}, \mathrm{Sm}, \mathrm{Nd}$, and $\mathrm{Yb}$ the magnetic moment of an orthoferrite experiences a $90^{\circ}$ reorientation transition in the temperature interval $\left(T_{2}, T_{1}\right)$. Upon lowering the temperature the magnetization continuously rotates from the crystalline $c$ axis at $T_{1}$ to the $a$ axis at $T_{2}$. The interest to the reorientation transition was renewed relatively recently by the paper [2] which focused on the precise measurements of the magnetization $M(T)$ and gave an explanation of the observed temperature dependence in the framework of the modified mean field theory.

As a side observation it was pointed out in Ref. 2 that the shapes of the hysteresis loops $M(H)$ were markedly different inside the reorientation region and outside of it. Outside of $\left(T_{2}, T_{1}\right)$ the hysteresis loops were rectangular, whilst inside this interval they developed triangular tails or even had two separate triangular sub-loops (see Fig. 4 in Ref. 2). The issue was not investigated further at the time, but later it was found [3] that the same types of hysteresis loops also occur in $\mathrm{ErFeO}_{3}$ at the temperatures $T<20 \mathrm{~K}$, far below the reorientation transition that occurs at $88-97 \mathrm{~K}$. The peculiar loop shapes were dubbed the «triangular tail» and «double triangle» loops. The explanation of the triangular loops was suggested in Ref. 4 based on the assumption of the extremely simple two-domain magnetic structure of the orthoferrite sample. The two-domain state of the relatively large $(3 \times 4 \times 4 \mathrm{~mm})$ sample was supported by the theoretical estimates and was due to the very small magnetization of the orthoferrite. The motion of the domain wall, and the corresponding size changes of the up and down domains, accounted for the magnetization jumps on the hysteresis loop.

Since the loop shapes at low temperatures and in the reorientation interval are similar, it is tempting to try to explain their shape by the same mechanism. The triangular loops are explained in Ref. 4 as a result of an interplay between the domain wall «expulsion field» $H_{\exp }$ and the domain wall nucleation field $H_{n}$. The former is the field at which the domain wall reaches the boundary of the sample and is expelled from it as the field is raised. The latter is

Wisdom overcomes difficulty.

(C) Ya.B. Bazaliy and L.T. Tsymbal, 2010 
the field at which the domain wall is nucleated in the sample as the field is lowered. The existence of the nucleation field reflects the fact that for a domain wall to enter the sample the system has to overcome a certain wall nucleation barrier. An applied field $H$ creates a «magnetic pressure» acting against this barrier and causing the wall to nucleate. The analysis of Ref. 4 shows that rectangular hysteresis loops correspond to the case $H_{n}<-H_{\text {exp }}$, triangular tail loops correspond to $-H_{\exp }<H_{n}<0$, and double-triangle loops - to $0<H_{n}<H_{\text {exp }}$. In the model of Ref. 4 the fields $H_{\exp }$ and $H_{n}$ are related by an equation

$$
H_{n}=H_{\text {exp }}-\frac{\text { const }}{M(T)} .
$$

This formula qualitatively explained the evolution of the loop shapes using the experimentally measured temperature dependence of the magnetization below $20 \mathrm{~K}$.

Consider now an experiment in the reorientation region. The field $H_{a}$ is applied along the $a$ axis and the temperature is raised from below into the reorientation interval. A formal application of (1) gives a result contradicting the experiments. Since $M$ decreases above $T_{2}$ [1,5], this formula suggests that $H_{n}$ should become even more negative. This in turn would mean that the hysteresis loops have to remain rectangular, rather than acquire the triangular shapes.

To explain the discrepancy between the theory and the experiment we note that above $T_{2}$ the anisotropy energy of the orthoferrite changes and thus the formulas obtained for the uniaxial anisotropy below $T_{2}$ have to be revised. To properly consider the wall nucleation in the reorientation region one has to take into account two circumstances. First, when magnetization $\mathbf{M}$ points at an angle to the $a$ axis the demagnetization energy changes. Second, in the reorientation region the crystalline anisotropy is not uniaxial. This changes the structure and energy of the domain walls and, as a result, can change the properties of the domain wall entrance barrier. The aim of the present paper is to take both effects into account.

The magnetization of an orthoferrite is a sum of the iron magnetization $\mathbf{F}(T)$ characterized by a constant absolute value and directed at an angle $\theta_{F}(T)$ with respect to the $c$ axis, and the temperature-dependent rare-earth magnetization $\mathbf{m}(T)$. The iron moments are ordered due to the interactions between them. The interaction between the rareearth ions is negligible, but they are magnetized by the molecular field of the iron moments. This situation can be modeled [2] by the free energy density

$$
\begin{aligned}
& E=\frac{1}{2} K_{u}(T) \cos \left(2 \theta_{F}\right)+K_{b} \cos \left(4 \theta_{F}\right)- \\
& -\beta\left(F_{a} \chi_{a} m_{a}+F_{c} \chi_{c} m_{c}\right)+\frac{\beta}{2} m^{2},
\end{aligned}
$$

where $K_{u, b}$ are the crystalline anisotropy constants with $K_{u}(T)$ changing linearly with temperature in the reorientation interval, the coefficients $\chi_{a, b}$ describe the susceptibilities of the rare-earth moments to the molecular field of iron ions, and $\beta$ characterizes the free energy of the paramagnetic system of rare-earth moments. Minimization with respect to $\mathbf{m}$ gives the desired paramagnetic behavior of the rare-earth system, $m_{i}=\chi_{i} F_{i}(i=a, c)$, and a reduced expression for the free energy

$$
E=\frac{1}{2} K_{u}^{\prime}(T) \cos \left(2 \theta_{F}\right)+K_{b} \cos \left(4 \theta_{F}\right)+\text { const },
$$

with $K_{u}^{\prime}=K_{u}-\beta F^{2}\left(\xi_{c}^{2}-\xi_{a}^{2}\right) / 2$. For $K_{b}>0$ this energy form provides the reorientation transition in the interval $-8 K_{b}<K_{u}^{\prime}(T)<8 K_{b}[1]$, where the $-8 K_{b}$ end of the interval corresponds to the high-temperature phase $\left(\theta_{F}=0\right.$, $\mathbf{F} \| c$ ) and the $+8 K_{b}$ end corresponds to the lowtemperature phase $\left(\theta_{F}=\pi / 2, \mathbf{F} \| a\right)$. The energy profile $E\left(\theta_{F}\right)$ in the reorientation region is shown in Fig. 1. Its maxima are located at $\theta_{F}=0, \pi / 2$ and its minimum point is found at the angle $\theta_{*}$ determined by the equation

$$
\cos \left(2 \theta_{*}\right)=-\frac{K_{u}^{\prime}}{8 K_{b}} .
$$

The four equilibrium directions are given by $\theta_{F}=$ $= \pm \theta_{*}, \pi \pm \theta_{*}$.

First, we study the domain wall properties in the reorientation region. Two types of domain walls are possible [6]. In the «a-wall» the angle changes between $\theta_{*}$ and $-\theta_{*}$, and in the «c-wall» it changes between $\theta_{*}$ and $\pi-\theta_{*}$. The free energy density of the $a$-wall starts from $E\left(\theta_{*}\right)$ in one domain, goes through the maximum value $E(0)$ and returns back to $E\left(\theta_{*}\right)$. In the $c$-wall the maximum free energy density if $E(\pi / 2)$. For definiteness, consider the $a$-wall which is nucleated in the experiment with $H \| a$ because it separates the domains that differ in magnetization projections $M_{a}$ on the field direction. We now want to estimate the width and energy of this wall. Assuming that the $(a, c)$ plane of magnetization rotation is
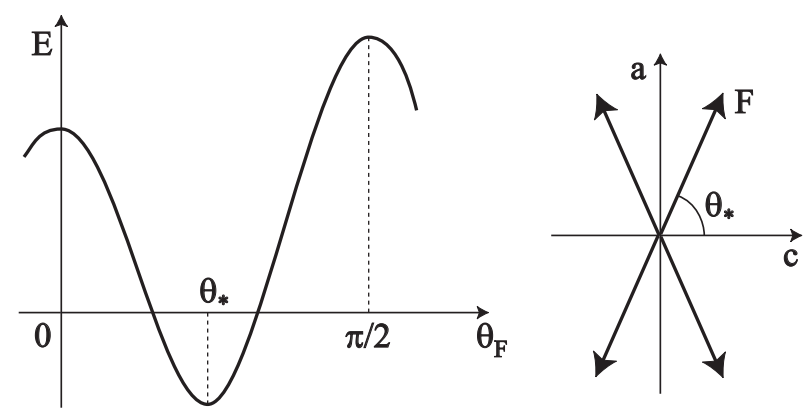

Fig. 1. Left: angular dependence of the free energy $E\left(\theta_{F}\right)$. Right: equilibrium directions of the iron magnetization $\mathbf{F}$. 
also the wall plane, i.e., considering a Bloch wall with zero demagnetization energy, we obtain the total free energy

$$
\mathcal{E}=\int E_{\text {tot }} d x=\int\left[\frac{J}{2}\left(\frac{d \theta_{F}}{d x}\right)^{2}+E\left(\theta_{F}\right)\right] d x,
$$

where $J$ is the spin stiffness, and $x$ is a coordinate along the $b$ axis perpendicular to the $(a, c)$ plane. The exact shape of the wall $\theta_{F}(x)$ can be determined from the equation $\delta E_{\text {tot }} / \delta \theta_{F}=0$ as it has been done in Ref. 6 , but instead we will estimate the properties of the wall as follows. Let the wall width be $\delta_{a}$. Then $\theta_{F}^{\prime} \approx 2 \theta_{*} / \delta_{a}$ and the gradient contribution to its energy can be estimated as $\sim J \theta_{*}^{2} / \delta_{a}$. The contribution of the crystalline energy inside the wall can be estimated as $\sim F(0) \delta_{a}$. The wall energy is the difference between the states with and without the wall

$$
\Delta \mathcal{E}_{\mathrm{tot}} \sim \frac{J \theta_{*}^{2}}{\delta_{a}}+\left[E(0)-E\left(\theta_{*}\right)\right] \delta_{a} .
$$

Minimizing it with respect to the wall width we find

$$
\delta_{a} \sim \theta_{*} \sqrt{\frac{J}{E(0)-E\left(\theta_{*}\right)}} .
$$

The free energy values at the extremum points can be calculated exactly as

$$
\begin{gathered}
E(0)=K_{b}+\frac{K_{u}^{\prime}}{2}, \quad E\left(\frac{\pi}{2}\right)=K_{b}-\frac{K_{u}^{\prime}}{2}, \\
E\left(\theta_{*}\right)=-K_{b}-\frac{K_{u}^{\prime 2}}{32 K_{b}},
\end{gathered}
$$

which gives $E(0)-E\left(\theta_{*}\right)=2 K_{b}\left(1+K_{u}^{\prime} / 8 K_{b}\right)^{2}$. The angle $\theta_{*}$ can be approximated from Eq. (4) as

$$
\theta_{*} \approx \frac{\pi}{4}\left(1+\frac{K_{u}^{\prime}}{8 K_{b}}\right) .
$$

Using these expressions we find

$$
\begin{gathered}
\delta_{a} \sim \sqrt{\frac{J}{K_{b}}} \\
\Delta \mathcal{E}_{D W}^{a} \sim \sqrt{J K_{b}}\left(1+\frac{K_{u}^{\prime}}{8 K_{b}}\right)^{2} .
\end{gathered}
$$

Figure 2 compares the exact result from Ref. 6 with the approximation that uses Eq. (9) and sets the numeric coefficient to $\pi / 2$

$$
\Delta \mathcal{E}_{D W}^{a} \approx \frac{\pi}{2} \sqrt{J K_{b}}\left(1+\frac{K_{u}^{\prime}}{8 K_{b}}\right)^{2}
$$

As one can see, expression (10) turns out to be quite accurate. The $a$-wall energy gradually decreases to zero as the temperature is raised from $T_{2}$ to $T_{1}$ and $K_{u}^{\prime}(T)$ changes from $+8 K_{b}$ to $-8 K_{b}$. Physically this happens because the

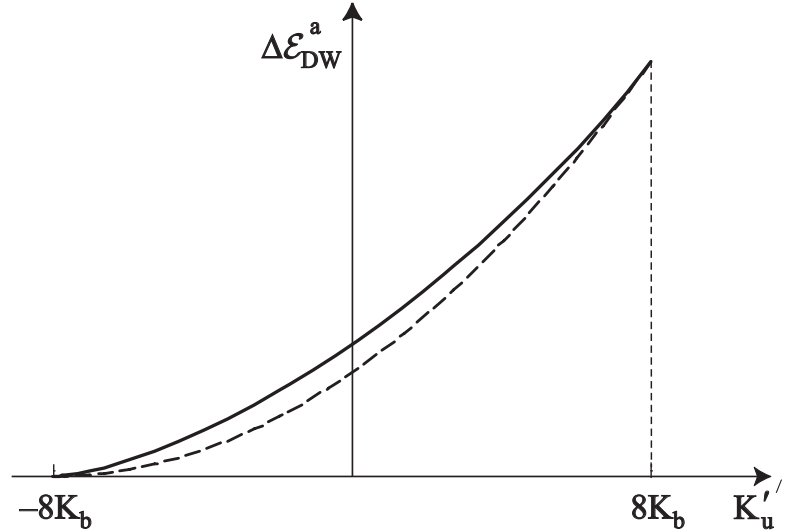

Fig. 2. Energy of the $a$-wall as a function of $K_{u}^{\prime}$. The solid line shows the exact dependence (Eq. (44) from Ref. 6). The dashed line is given by the approximation (10).

magnetization directions in two domains get closer to each other until the difference between them disappears at $T_{1}$.

The same calculation for the $c$-wall gives $\delta_{c}=\delta_{a}$ and

$$
\Delta \mathcal{E}_{D W}^{c} \approx \frac{\pi}{2} \sqrt{J K_{b}}\left(1-\frac{K_{u}^{\prime}}{8 K_{b}}\right)^{2} .
$$

Next, we consider the demagnetization energy $E_{d}$. A rectangular sample with an $a$-wall in it is shown in Fig. 3. The faces of the rectangle are assumed to be cut perpendicular to the crystal axis. We will treat the dipole-dipole energy as the interaction energy of the surface magnetic charges on the faces of the sample [7]. For the domain walls considered here the magnetic charges exist on the $a$ and $c$-faces (i.e., faces perpendicular to the $a$ and $c$ axis). The demagnetization energy can be divided into three parts: a contribution from the interaction between the charges on $a$-faces, between the charges on the $c$-faces, and

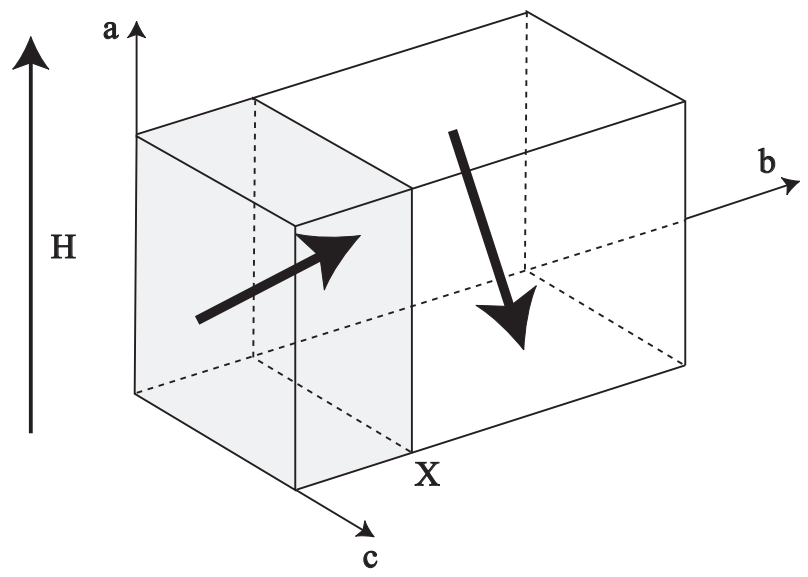

Fig. 3. Two-domain magnetic structure of a rectangular sample. Thick arrows show the magnetization directions inside the domains that are separated by an $a$-wall located at $X$. 
a cross-term from the interaction between the $a$-faces and $c$-faces. Since the energy is proportional to the product of the charges, one can write

$$
E_{d}=d_{a a} M_{a}^{2}+d_{c c} M_{c}^{2}+d_{a c} M_{a} M_{c}
$$

where the coefficients $d_{a a}, d_{c c}$ and $d_{a c}$ reflect the dimensions of the sample and the position of the domain wall in it. Note that the dipole energy depends on the full magnetic moment with $M_{a}=\left(1+\chi_{a}\right) F \sin \theta_{F}$ and $M_{c}=\left(1+\chi_{c}\right) F \cos \theta_{F}$. The cross-term turns out to be zero by symmetry, $d_{a c}=0$. Indeed, it is obvious from Fig. 3 that the interaction of magnetic charges on the upper $a$-face of the sample with charges on each $c$-face is exactly compensated by the interaction of the opposite charges on the lower $a$-face with the same $c$-face.

Similar to Ref. 4 we will approximate the demagnetization energy dependence on the position of the domain wall by a quadratic function. The position of the wall will be given by a dimensionless coordinate $X$ with $X \in[-1,1]$, and we will write $E_{d}(X)=E_{d}^{(0)}+D X^{2}$. For an $a$-wall the charges on the $c$-faces do not depend on the position of the wall in the sample. According to the argument above this means $D \sim M_{a}^{2}$, so that

$$
E_{d a}(X)=E_{d a}^{(0)}+A M_{a}^{2} X^{2}
$$

where the coefficient $A$ accounts for the shape and size of the sample. By the same argument $E_{d c}(X)=E_{d c}^{(0)}+$ $+C M_{c}^{2} X^{2}$ for the $c$-wall.

We can now finally proceed to the calculation of the expulsion and nucleation fields. The total energy of the sample with an $a$-wall is [4]

$$
\mathcal{E}(X)=\mathrm{const}+A M_{a}^{2} x^{2}-M_{a} H_{a} x+U_{D W}(X),
$$

where $U_{D W}(X)$ is the position-dependent energy of the domain wall. For $X$ well inside the sample it is a constant $U_{D W}=\Delta \mathcal{E}_{D W}^{a}$. However, when the wall approaches the sample boundary, the energy $U_{D W}(X)$ becomes essentially position-dependent. In the absence of an extra surface pinning, $U_{D W}(X)$ drops from $\Delta \mathcal{E}_{D W}^{a}$ to zero on the distance of the domain wall width $\delta_{a}$. A sketch of the free energy $\mathcal{E}(X)$ is shown in Fig. 4.

The expulsion field $H_{\text {exp }}$ is obtained from (13) using the condition $X= \pm 1$. This gives

$$
H_{\text {exp }}= \pm 2 A M_{a} \text {. }
$$

The nucleation field is determined from the condition [4] $d \mathcal{E} /\left.d X\right|_{x= \pm 1}=0$, where the derivative $d U_{D W} / d x$ can be estimated as $\Delta \mathcal{E}_{D W}^{a} / \delta_{a}$ (Fig. 4). This gives

$$
H_{n a}=H_{\text {exp }}-\frac{E(0)-E\left(\theta_{*}\right)}{M_{a}\left(\theta_{*}\right)} .
$$

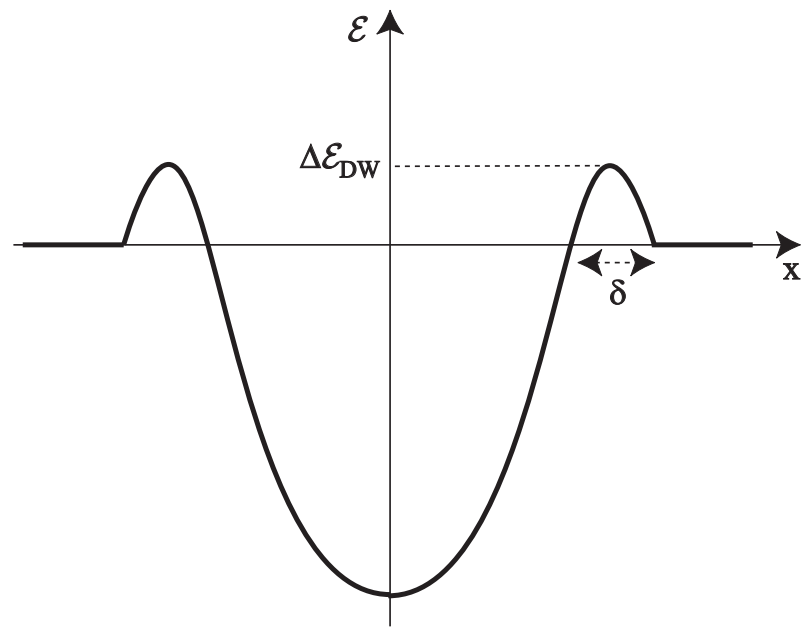

Fig. 4. Sketch of the domain wall energy position dependence. The nucleation barrier is determined by the wall energy and width.

Using the expression for $M_{a}\left(\theta_{F}\right)$ and Eq. (4) for $\theta_{*}$ one finds $M_{a}=\left(1+\chi_{a}\right) F \sqrt{\left(1+K_{u}^{\prime} / 8 K_{b}\right) / 2}$. This gives

$$
H_{\text {exp }}-H_{n a} \sim \frac{K_{b}}{\left(1+\chi_{a}\right) F_{a}}\left(1+\frac{K_{u}^{\prime}}{8 K_{b}}\right)^{3 / 2} .
$$

This equation is the main result of the paper. After taking a proper account of the change of anisotropy in the reorientation region we have found that the difference $H_{\exp }-H_{n a}$ decreases as the temperature rises above $T_{2}$. The behavior of both fields is sketched in Fig. 5. While the value of $M_{a}$ in the denominator of (15) does decrease as discussed in the introduction, the domain wall energy and the corresponding nucleation barrier decrease faster, leading to a theoretical prediction that is in a qualitative agreement with the experimental findings: as $H_{n}$ approaches $H_{\text {exp }}$,

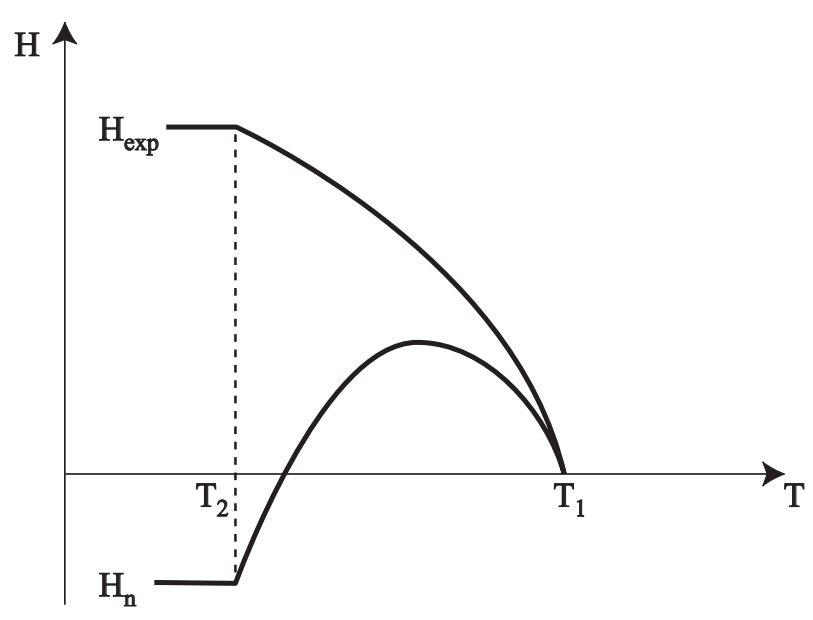

Fig. 5. Temperature dependence of the expulsion field $H_{\exp }$ and nucleation field $H_{n}$ in an experiment with $H \| a$. 
the loop shape changes from rectangular to triangular tails and then to a double-triangle loop.

For the $c$-wall the same arguments give the relationship

$$
H_{\mathrm{exp}}-H_{n c} \sim \frac{K_{b}}{\left(1+\chi_{c}\right) F_{c}}\left(1-\frac{K_{u}^{\prime}}{8 K_{b}}\right)^{3 / 2},
$$

from which one concludes that in a measurement with $H \| c$ and temperature decreasing from above into the reorientation region, the hysteresis loop shape will change from a rectangular to a triangular due to the nucleation of a $c$-wall. Such a prediction is again in accord with the experiments.

Our theory also makes predictions about the hysteresis loop shape in the case of magnetic field tilted in the $(a, c)$ plane. Here either an $a$-wall, or a $c$-wall, or both, can be nucleated, depending on the values of $H_{n a, n c}$ and the projections of the applied field $H_{a, c}$. For the temperature values at which the nucleation field $H_{n a}$ lies in the interval $\left(-H_{\exp , a}, H_{\exp , a}\right)$, and the nucleation field $H_{n c}$ lies in the interval $\left(-H_{\exp , c}, H_{\exp , c}\right)$, one should observe two jumps on the downward and upward branches of the hysteresis loop. These jumps will correspond to the nucleation of $a$ - and $c$-domain walls.
In conclusion, we have shown that the temperature dependence of the domain wall structure in the magnetic reorientation interval can qualitatively explain the evolution of the hysteresis loop shapes in orthoferrites. We further suggested that a measurement in a tilted magnetic field can serve as an experimental check of our theory.

Ya.B. was partially supported by the NSF grant DMR0847159 .

1. K.P. Belov, A.K. Zvezdin, A.M. Kadomtseva, and R.Z. Levitin, Orientation Phase Transitions in Rare Earth Magnetic Materials, Nauka, Moscow (1979) (in Russian).

2. Y.B. Bazaliy, L.T. Tsymbal, G.N. Kakazei, A.I. Izotov, and P.E. Wigen, Phys. Rev. B69, 104429 (2004).

3. L.T. Tsymbal, Y.B. Bazaliy, G.N. Kakazei, F.J. Palomares, and P.E. Wigen, IEEE Trans. Magn. 44, 2933 (2008).

4. L.T. Tsymbal, G.N. Kakazei, and Y.B. Bazaliy, Phys. Rev. B79, 092414 (2009).

5. R.L. White, J. Appl. Phys. 40, 1061 (1969).

6. V.G. Bar'yakhtar, B.A. Ivanov, and A.L. Sukstanskii, ZhETF 78, 1509 (1980) [Sow. Phys. JETP 51, 757 (1980)].

7. M.E. Schabes and A. Aharoni, IEEE Trans. Magn. 23, 3882 (1987). 